\title{
NORM OF A DERIVATION ON A VON NEUMANN ALGEBRA $\left({ }^{1}\right)$
}

\author{
BY \\ P. GAJENDRAGADKAR
}

\begin{abstract}
A derivation on an algebra $\mathfrak{U}$ is a linear function $\mathscr{D}: \mathfrak{U} \rightarrow \mathfrak{U}$ satisfying $\mathscr{D}(a b)=\mathscr{D}(a) b+a \mathscr{D}(b)$ for all $a, b$ in $\mathfrak{U}$. If there exists an $a$ in $\mathfrak{U}$ such that $D(b)=a b-b a$ for $b$ in $थ$, then $D$ is called the inner derivation induced by $a$. If $थ$ is a von Neumann algebra, then by a theorem of Sakai [7], every derivation on $\mathscr{U}$ is inner. In this paper we compute the norm of a derivation on a von Neumann algebra. Specifically we prove that if $\ell$ is a von Neumann algebra acting on a separable Hilbert space $\mathcal{H}, T$ is in $\mathscr{U}$, and $\mathscr{D}_{T}$ is the derivation induced by $T$, then $\left\|D_{T} \mid \mathfrak{\imath}\right\|=2 \inf \{\|T-Z\|, Z$ in centre $\mathfrak{U}\}$.
\end{abstract}

In this paper we answer one of the questions raised by J. Stampfli in [8], viz. computing the norm of a derivation on an arbitrary $C^{*}$-algebra. It follows from [3, Lemma 3] that a derivation on a $C^{*}$-algebra acting on a Hilbert space $\mathcal{H}$ can be extended to its weak-closure in $\mathscr{B}(\mathcal{H})$, the algebra of all bounded operators acting on $\mathcal{H}$. Kaplansky's density theorem proves that this extension is achieved without increasing the norm. So there is no loss of generality in restricting our attention to von Neumann algebras. This result when $T$ is selfadjoint has been proved by Kadison, Lance and Ringrose. But the methods have little in common.

1. Preliminaries. We shall use decomposition of a Hilbert space into a direct integral relative to a given abelian von Neumann algebra. We quote two theorems for future reference and refer the reader to [6] for notations and proofs.

Theorem. To every commutative von Neumann algebra $\mathcal{C}$ on a separable Hilbert space $\mathcal{H}$ there corresponds a decomposition of $\mathcal{H}$ into direct integral with $\mathcal{C}$ as the totality of all operators of the form $L_{\phi}$.

Theorem. Under the same bypothes is as in the previous theorem, for an arbitrary family $\mathfrak{U} \subseteq \mathcal{C}^{\prime}$, every element $A$ in $\mathfrak{U}$ is decomposable. Let $\mathfrak{U}(\lambda)=$ $\{A(\lambda), A$ in $\mathscr{Q}\}$. Then the family $\mathfrak{U}(\lambda)$ is irreducible for almost all $\lambda$ if and only if $\mathcal{C}$ is a maximal abelian subalgebra of ' $\mathrm{U}^{\prime}$.

In [8], Stampfli has defined the centre of a bounded operator $T$ as the unique

Presented to the Society June 20,1970; received by the editors October 29, 1970.

AMS 1969 subject classifications. Primary 6703.

Key words and phrases. Centre of a bounded operator, decomposition of a Hilbert space into a direct integral, norm of a derivation, separable, von Neumann algebra.

(1) This paper formed a part of the author's dissertation for Ph.D. at Indiana University, under the guidance of Professor J. G. Stampfli. The research was partially supported by the National Science Foundation under Research Grants NSF GP 7505 and NSF 11734. 
complex number $\mu$ for which $\|T-\mu\|=\inf \{\|T-\lambda\|, \lambda$ a complex number $\}$. Following [8] we denote the centre of a bounded operator $T$ by $C_{T}$, centre of an algebra $\mathfrak{U}$ by $\mathscr{Z}(\mathfrak{U})$, and the complex numbers by $\mathrm{C}$. We assume that all Hilbert spaces are separable.

2. Lemma 1. If $\mathcal{U}$ is a von Neumann algebra acting on a Hilbert space $\mathcal{H}$ and if $T$ is in $\mathscr{U}$, then there exists $Z_{0}$ in $\mathscr{Z}(\mathfrak{U})$ such that, for every projection $P$ in $\mathcal{Z}(\mathfrak{Q}),\left\|\left(T-Z_{0}\right) P\right\|=\inf \{\|(T-Z) P\|, Z \in \mathcal{Z}(\mathfrak{Q})\}$.

Proof. First we note that if $a=\inf \{\|T-Z\|, Z \in \mathcal{Z}(\mathscr{Z})\}$, then there exists a sequence $\left\{Z_{n}\right\} \subseteq \mathcal{Z}(\mathscr{U})$ such that $\left\|Z_{n}-T\right\| \rightarrow \alpha$. Also, $\left\|Z_{n}\right\| \leq\left\|T-Z_{n}\right\|+\|T\|$ implies that $\left\{\left\|Z_{n}\right\|\right\}$ is a bounded sequence. Hence there exists a subsequence $\left\{Z_{n_{k}}\right\}$ such that $Z_{n_{k}} \rightarrow Z_{0}$ in the weak operator topology. It follows that $Z_{0} \epsilon$ $\mathcal{Z}(\mathfrak{L})$ and further,

$$
\left\|T-Z_{0}\right\| \leq \underline{\lim }\left\|T-Z_{n_{k}}\right\|=\alpha .
$$

Thus $\left\|T-Z_{0}\right\|=\alpha$.

Now if $P$ is a projection in $\mathcal{Z}(\mathscr{U})$, applying the previous case to the algebra $\mathfrak{U}_{P}$ and operator $T P$, it follows that there exists $Z_{P} \in \mathscr{Z}(\mathscr{U})$ such that $\left\|\left(T-Z_{P}\right) P\right\|$ $=\inf \{\|(T-Z) P\|, Z \in \mathscr{Z}(\mathfrak{U})\}$.

If $\left\{P_{i}\right\}_{i=1}^{n}$ is a family of pairwise orthogonal projections in $\mathcal{Z}(\mathscr{U})$ with $\sum_{i=1}^{n} P_{i}=I$, we know that there exist $\left\{Z_{i}\right\}_{i=1}^{n} \subseteq \mathcal{Z}(\mathcal{Q})$ satisfying $\left\|\left(T-Z_{i}\right) P_{i}\right\|=$ $\inf \left\{\left\|(T-Z) P_{i}\right\|, Z \in \mathcal{Z}(\mathscr{Q})\right\}, 1 \leq i \leq n$. Define $Z^{1}=\sum_{i=1}^{n} Z_{i} P_{i}$ and denote $Z^{1}=$ $Z_{\left\{P_{1}, \cdots, P_{n}\right\}}$ Then $\left\|\left(T-Z^{1}\right) P_{i}\right\|=\inf \left\{\left\|(T-Z) P_{i}\right\|, Z \in \mathcal{Z}(\mathfrak{Q})\right\}$ for $1 \leq i \leq n$.

Let $\mathcal{F}^{n}$ be the class of all finite orthogonal families $\left\{Q_{1}, \ldots, Q_{n}\right\}$ with sum $I$. We say $\left\{P_{1}, \ldots, P_{m}\right\} \leq\left\{Q_{1}, \ldots, Q_{n}\right\}$ if each $P_{i}$ is a sum of a subset of $Q$ 's. Note that if $\left\{Q_{1}, \cdots, Q_{n}\right\} \in \mathcal{F}$, then

$$
\left\|T-Z_{\left\{Q_{1}, \cdots, Q_{n}\right\}}\right\|=\inf \{\|T-Z\|, Z \text { in } \mathcal{Z}(\mathscr{Q})\} .
$$

It follows that $\left\{Z_{\left\{Q_{1}, \cdots, Q_{n}\right\}},\left\{Q_{1}, \cdots, Q_{n}\right\} \in \mathcal{F}\right\}$ is a bounded net. Hence there is a cofinal subset which converges to $Z_{0}$ in $Z(\mathscr{Z})$ in weak-operator-topology. We now prove that, for any projection $Q$ in $\mathscr{Z}(\mathscr{L})$,

$$
\left\|\left(T-Z_{0}\right) Q\right\|=\inf \{\|(T-Z) Q\|, Z \text { in } \mathcal{Z}(\mathscr{U})\} .
$$

For if $Q$ is a projection in $\mathcal{Z}(\mathcal{Q}),\{Q, I-Q\} \in \mathcal{F}$, and if $\left\{Q_{1}, \cdots, Q_{n}\right\} \geq\{I-Q, Q\}$, we may write (with possible renumbering) $Q=Q_{1}+\cdots+Q_{k}, k \leq n$.

$$
\begin{aligned}
\left\|\left(T-Z_{\left\{Q_{1}, \cdots, Q_{n}\right\}}\right\} Q\right\| & =\sup _{1 \leq i \leq k}\left\|\left(T-Z_{\left\{Q_{1}, \cdots, Q_{n}\right\}}\right) Q_{i}\right\| \\
& \leq \sup _{1 \leq i \leq k}\left\|(T-Z) Q_{i}\right\| \text { for all } Z \text { in } \mathcal{Z}(\mathscr{Q}) .
\end{aligned}
$$


Thus $\left\|\left(T-Z_{\left\{Q_{1}, \cdots, Q_{n}\right\}}\right) Q\right\| \leq\|(T-Z) Q\|$ for all $Z \in \mathcal{Z}(\mathcal{U})$ and $\left\{Q_{1}, \cdots, Q_{n}\right\} \geq$ $\{Q, I-Q\}$.

Since weak limits do not increase norm,

$$
\left\|\left(T-Z_{0}\right) Q\right\| \leq\|(T-Z) Q\| \text { for all } Z \text { in } \mathcal{Z}(\mathcal{Q}) \text {. }
$$

Lemma 2. Let $\mathcal{H}=\int^{\oplus} \mathcal{H}_{\lambda} d \sigma(\lambda)$ and $T$ in $\mathfrak{B}(\mathcal{H})$ be a decomposable operator, i.e. $T=T(\lambda)$ where $\{T(\lambda)\}$ is an essentially bounded, measurable operator function. Then the scalar-valued function $C_{T(\lambda)}$ is essentially bounded and measurable.

Proof. Let $\mathcal{H}=\int^{\oplus} \mathcal{H} \lambda_{\lambda} d \sigma(\lambda)$ and let $T$ in $\mathscr{B}(\mathcal{H})$ be written as $T=\{T(\lambda)\}$. Let $\mathscr{Z}=\left\{L_{\phi}, \phi \in L^{\infty}(\Lambda, \sigma)\right\}$ and $\mathscr{U}=\mathscr{Z}^{\prime}=$ the algebra of all decomposable operators on $\mathcal{H}$.

Then $T \in \mathscr{U}$ and $\mathscr{Z}(\mathscr{Z})=\mathscr{U} \cap \mathfrak{U}^{\prime}=\mathscr{Z}^{\prime} \cap \mathscr{Z}=\mathscr{Z}$. By Lemma 1 , there exists $Z_{0}=$ $L_{\phi}$ in $\mathcal{Z}$ such that, for every projection $P$ in $\mathcal{E}$,

$$
\left\|\left(T-Z_{0}\right) P\right\|=\inf \{\|(T-Z) P\|, Z \in \mathcal{Z}\} .
$$

We assert that $\phi(\lambda)=C_{T(\lambda)}$ a.e.

If $\mu \in \mathbf{C}$ and $\epsilon>0$, let

$$
E=\left\{\lambda \in \Lambda,\left\|T(\lambda)-\mu_{\lambda}\right\| \leq\left\|T(\lambda)-\phi(\lambda) I_{\lambda}\right\|-\epsilon\right\}
$$

and $\psi_{E}$ denote the characteristic function of $E$. Then $Z_{1}=\left\{\mu I_{\lambda}\right\} \in \mathcal{Z}$ and $P=$ $\left\{\psi_{E}(\lambda) I_{\lambda}\right\}$ is a projection in $\mathcal{Z}$. Since $\left\|\left(T-Z_{0}\right) P\right\| \leq\left\|\left(T-Z_{1}\right) P\right\|$, it follows that $\sigma(E)=0$. If $\left\{\mu_{n}\right\}_{1}^{\infty}$ is a dense subset of complex numbers, write

$$
F=\bigcup_{n=1}^{\infty}\left\{\lambda \in \Lambda,\left\|T(\lambda)-\mu_{n} I_{\lambda}\right\|<\left\|T(\lambda)-\phi(\lambda) I_{\lambda}\right\|\right\} .
$$

It follows that $\sigma(F)=0$.

If $\lambda \notin F$ and $\mu \in \mathbf{C}, \mu_{n_{k}} \rightarrow \mu$ for some subsequence $\left\{n_{k}\right\}$, and

$$
\left\|T(\lambda)-\mu I_{\lambda}\right\|=\lim _{k \rightarrow \infty}\left\|T(\lambda)-\mu_{n_{k}} I_{\lambda}\right\| \geq\left\|T(\lambda)-\phi(\lambda) I_{\lambda}\right\| \text {. }
$$

By uniqueness of $C_{T(\lambda)}$, it follows that, for $\lambda \notin F, C_{T(\lambda)}=\phi(\lambda)$. Since $\sigma(F)=$ 0 , this proves the claim and consequently the lemma.

Corollary 1. The element $Z$ obtained in Lemma 1 is unique, when $\mathscr{A}$ is a type I algebra.

Theorem 1. Let $\mathfrak{U}$ be a von Neumann algebra on $\mathcal{H}$ and assume that $\mathfrak{U}^{\prime}$ is abelian. Then for $T$ in $\mathfrak{U}$, there exists $Z_{0}$ in $\mathcal{Z}(\mathfrak{U})=\mathfrak{U}^{\prime}$ such that $\left\|\mathscr{D}_{T} \mid \mathfrak{U}\right\|=$ $2\left\|T-Z_{0}\right\|$.

Proof. Let $\mathcal{H}=\int_{\lambda}^{\oplus} \mathcal{H}_{\lambda} d \sigma(\lambda)$ be the decomposition corresponding to the 
abelian algebra $\mathscr{Z}(\mathfrak{U})=\mathscr{U} \mathfrak{X}^{\prime}$. Then $\mathscr{Z}(\mathfrak{Z})=\left\{L_{\phi}, \phi \in L^{\infty}(\Lambda, \sigma)\right\}$ and $\mathfrak{X}=$ the family of all decomposable operators on $\mathcal{H}$. Also there exists a $\sigma$-null set $F$ such that $\lambda \notin F$ implies $\mathscr{U}(\lambda)$ is an irreducible algebra. Define $\phi(\lambda)=C_{T(\lambda)}$ if $\lambda \notin F$ and $\phi(\lambda)=0$ if $\lambda \in F$. Then by Lemma $2, \phi \in L^{\infty}(\Lambda, \sigma)$ and hence $L_{\phi} \in \mathcal{Z}(\mathcal{Z})$. By Stampfli's theorem [8], for $\lambda \notin F,\left\|D_{T(\lambda)} \mid \mathcal{P}(\lambda)\right\|=2\left\|T(\lambda)-C_{T(\lambda)} I_{\lambda}\right\|$ and thus

$$
2\left\|T-L_{\phi}\right\|=2 \text { ess } \sup _{\lambda}\left\|T(\lambda)-C_{T(\lambda)} I_{\lambda}\right\|=\underset{\lambda}{\operatorname{sss} \sup }\left\|\mathscr{D}_{T(\lambda)} \mid \mathfrak{U}(\lambda)\right\| \text {. }
$$

We prove that ess sup $\left\|\mathscr{D}_{T(\lambda)}\left|\mathfrak{Q}(\lambda)\|\leq\| \mathscr{D}_{T}\right| \mathfrak{A}\right\|$.

We set $\Lambda_{k}=\left\{\lambda \in \Lambda, \operatorname{dim} \mathcal{H}_{\lambda}=k\right\}$. Then $\Lambda=\bigcup_{k} \Lambda_{k}$ and it is sufficient to prove that

$$
\sigma\left\{\lambda \in \Lambda_{k},\left\|\mathscr{D}_{T(\lambda)}\right\|>\left\|\mathscr{D}_{T} \mid \mathfrak{A}_{\|}\right\|=0 \quad \text { for all } k .\right.
$$

Fix $k$ for which $\sigma\left(\Lambda_{k}\right) \neq 0$ and write $\mathcal{H}_{k}=\int_{\Lambda_{k}}^{\oplus} \mathcal{H}_{\lambda} d \sigma(\lambda)$ where all $\mathcal{H}_{\lambda}$ can be identified with the same Hilbert space $\mathcal{K}$. Let $\left\{B_{n}, n \geq 1\right\}$ be a sequence which is weakly dense in the unit ball of $\mathscr{B}(\mathcal{K})$. Defining $A_{n}(\lambda)=B_{n}$ for $\lambda \in \Lambda_{k}$ and $A_{n}(\lambda)=0$ for $\lambda \notin \Lambda_{k}$, we see that $A_{n}=A_{n}(\lambda)$ is an essentially bounded, measurable, operator function and hence $A_{n}^{n} \in \mathscr{P}^{n}(\mathcal{H})$ with $\left\|A_{n}\right\| \leq 1$. Since by definition $A_{n}$ is a decomposable operator, $A_{n} \in \mathcal{U l}$. Now

$$
\begin{aligned}
\underset{\lambda \in \Lambda_{k}}{\text { ess } \sup _{n}}\left\|B_{n} T(\lambda)-T(\lambda) B_{n}\right\| & =\underset{\lambda \in \Lambda}{\text { ess } \sup _{n}}\left\|A_{n}(\lambda) T(\lambda)-T(\lambda) A_{n}(\lambda)\right\| \\
& =\left\|A_{n} T-T A_{n}\right\| \leq\left\|D_{T} \mid \mathcal{Q}\right\| .
\end{aligned}
$$

Hence if we write $E_{n}=\left\{\lambda \in \Lambda_{k},\left\|T(\lambda) B_{n}-B_{n} T(\lambda)\right\|>\left\|\mathscr{D}_{T} \mid \mathfrak{P}\right\|\right\}$ and $E=\bigcup_{n=1}^{\infty} E_{n}$, we see that $\sigma\left(E_{n}\right)=0$ and hence $\sigma(E)=0$.

Next, we will show that, for $\lambda \in \Lambda_{k}, \lambda \notin E$,

$$
\left\|\mathscr{D}_{T(\lambda)}\left|\mathfrak{U}(\lambda)\|\leq\| \mathscr{D}_{T}\right| \mathfrak{Q}\right\| .
$$

Fix $\lambda_{0} \in \Lambda_{k}, \lambda_{0} \notin E$ and let $A \in \mathscr{U}$ with $\left\|A\left(\lambda_{0}\right)\right\| \leq 1$. Then there exists a subsequence $\left\{B_{n_{k}}\right\}$ (depending on $\lambda_{0}$ ), such that $B_{n_{k}} \rightarrow A\left(\lambda_{0}\right)$ in weak-operator-topology. Hence

$$
\left\|\mathscr{D}_{T}(A)\left(\lambda_{0}\right)\right\| \leq \underline{\lim }\left\|B_{n_{k}} T\left(\lambda_{0}\right)-T\left(\lambda_{0}\right) B_{n_{k}}\right\| \leq\left\|\mathscr{D}_{T} \mid \mathfrak{R}\right\| .
$$

Since $\mathscr{D}_{T}(A)\left(\lambda_{0}\right)=\mathscr{D}_{T\left(\lambda_{0}\right)} A\left(\lambda_{0}\right)$ we have proved that

$$
\left\|\mathscr{D}_{T(\lambda)}\left|\mathfrak{X}(\lambda)\|\leq\| \mathscr{D}_{T}\right| \mathfrak{Z}\right\| \quad \text { for all } \lambda \in \Lambda_{k}, \lambda \notin E .
$$

Thus ess $\sup _{\lambda}\left\|\mathscr{D}_{T(\lambda)}\left|\mathfrak{A}(\lambda)\|\leq\| \mathscr{D}_{T}\right| \mathfrak{A}\right\|$. Hence

$$
2\left\|T-L_{\phi}\right\|=\underset{\lambda}{\operatorname{ess} \sup }\left\|\mathscr{D}_{T(\lambda)}\left|\mathfrak{Q}(\lambda)\|=\| \mathscr{D}_{T}\right| \mathfrak{2}\right\| \leq 2\left\|T-L_{\phi}\right\|
$$


and

$$
2\left\|T-L_{\phi}\right\|=\left\|\mathfrak{D}_{T} \mid \mathfrak{Q}\right\| \text {. }
$$

Corollary 2. If $\mathcal{U}$ is a von Neumann algebra with $\mathfrak{U}^{\prime}$ abelian and $T$ is in $\mathscr{A}$, then there exists $Z_{0}$ in $\mathscr{Z}(\mathfrak{U})$ such that, for every projection $P$ in $\mathscr{Z}(\mathfrak{U})$,

$$
\left\|\mathfrak{D}_{T P} \mid \mathfrak{Q} P\right\|=2\left\|\left(T-Z_{0}\right) P\right\| \text {. }
$$

Proof. Let $P$ be a projection in $\mathscr{Z}(\mathfrak{U})=\mathfrak{U} \mathfrak{U}^{\prime}$. Consider the algebra $\mathfrak{U} P$ and $S=T P$. Since $(\mathscr{U} P)^{\prime}=\mathscr{U}^{\prime} P,(\mathscr{U} P)^{\prime}$ is abelian.

Since $P$ is a projection in $\mathscr{Z}(\mathscr{U}), P=L_{\eta}$, where $\eta$ is the characteristic function of a measurable set $E$. By Theorem 1 , we have

$$
\left\|\mathscr{D}_{S} \mid \mathfrak{U} P\right\|=2\left\|S-L_{\phi}\right\| \text { where } \psi(\lambda)=C_{S(\lambda)} \text { a.e. }
$$

It follows that $C_{S(\lambda)}=\eta(\lambda) C_{T(\lambda)}$ and hence

$$
\left\|\mathscr{D}_{S} \mid \mathfrak{U} P\right\|=2\left\|S-L_{\psi}\right\|=2 \text { ess } \sup _{\lambda}\left\|\left(T(\lambda)-C_{T(\lambda)}\right) \eta(\lambda)\right\|=2\left\|\left(T-Z_{0}\right) P\right\| .
$$

Theorem 2. Let $\mathfrak{U}$ be a von Neumann algebra on $\mathcal{H}$, and let $T$ be in $\mathcal{U}$. Then there exists $\xi$ in $\mathfrak{U}^{\prime}$ such that for every projection $P$ in $\mathcal{Z}(\mathfrak{U}),\left\|\mathscr{D}_{T P} \mid \mathfrak{U} P\right\|=$ $2\|(T-\xi) P\|$.

Proof. Let $\mathfrak{U}_{1}$ be the type I algebra containing $\mathfrak{U}$, as constructed in [3, p. 283]. Then by Kadison's argument, $\left\|\mathscr{D}_{T}\left|\mathfrak{U}\|=\| \mathscr{D}_{T}\right| \mathfrak{U}_{1}\right\|$. Moreover, applying the same argument to $\mathscr{U} P$ in place of $\mathscr{U}$, we see that, for any central projection $P$ in U, $\left\|\mathscr{D}_{T P}\left|\mathscr{U}_{P}\|=\| \mathscr{D}_{T P}\right| \mathfrak{U}_{1} P\right\|$. By Theorem 1 , there exists an element $\xi$ in $\mathscr{Z}\left(\mathscr{U}_{1}\right)$ such that $\left\|\mathscr{D}_{T Q} \mid \mathscr{X}_{1} Q\right\|=2\|(T-\xi) Q\|$ for every central projection $Q$ in $\mathscr{U}$. Since $\mathcal{Z}(\mathfrak{U}) \subseteq \mathcal{Z}\left(\mathfrak{U}_{1}\right) \subseteq \mathfrak{U}^{\prime}$, this completes the proof.

Theorem 3. Let $\mathcal{U}$ be a von Neumann algebra on a separable Hilbert space and let $T$ be in $\mathscr{U}$. Then there exists a unique $Z_{0}$ in $\mathcal{Z}(\mathfrak{U})$ such that, for every projection $P$ in $\mathscr{E}(\mathfrak{U}),\left\|\mathscr{D}_{T P} \mid \mathfrak{U} P\right\|=2\left\|\left(T-Z_{0}\right) P\right\|$.

Proof. By Theorem 2, there exists $\xi$ in $2 X^{\prime}$ such that, for every projection $P$ in $\mathscr{Z}(\mathfrak{C}),\left\|\mathscr{D}_{T P} \mid \mathfrak{Q} P\right\|=2\|(T-\xi) P\|$. Let $\mathcal{K}_{\xi}=\left\{\Sigma_{i=1}^{n} \lambda_{i} \mathcal{U}_{i} \xi \mathcal{U}_{i}^{*}, \lambda_{i} \geq 0, \Sigma \lambda_{i}=1\right.$, $\mathcal{U}_{i}$ unitary in $\left.\mathfrak{X}^{\prime}\right\}$, and $\mathcal{K}_{\xi}^{=}$, the norm closure of $\mathcal{K}_{\xi}$. If $\xi^{\prime}$ is in $\mathcal{K}_{\xi}$, and $P$ is a projection in $\mathscr{Z}(\mathfrak{Q})$,

$$
\left(T-\xi^{\prime}\right) P=\sum_{i=1}^{n} \lambda_{i}\left(T-U_{i}^{*} \xi \mathcal{U}_{i}\right) P=\sum_{i=1}^{n} \lambda_{i} \mathcal{U}_{i}^{*}(T P-\xi P) \mathcal{U}_{i}
$$

Hence $\left\|\left(T-\xi^{\prime}\right) P\right\| \leq\|(T-\xi) P\|$.

Since $\left\|\mathscr{D}_{T P}\left|\mathscr{U}_{P}\|\leq 2\|\left(T-\xi^{\prime}\right) P\|\leq 2\|(T-\xi) P\|=\| \mathscr{D}_{T P}\right| \mathscr{U}_{P}\right\|$, we have $\left\|\mathfrak{D}_{T P} \mid \mathfrak{U} P\right\|=2\left\|\left(T-\xi^{\prime}\right) P\right\|$ for all $\xi^{\prime} \in \bar{K}_{\xi}$.

By continuity, the same equality holds for all $\xi^{\prime}$ in $\mathcal{K}_{\xi}^{=}$. By $[1$, Theorem 1 , 
p. 272], $\mathcal{K}_{\xi}^{=} \cap \mathcal{Z}\left(\mathfrak{U}^{\prime}\right) \neq \varnothing$. Hence if $Z_{0}$ in $\mathcal{K}_{\xi}^{=} \cap \mathcal{Z}(\mathfrak{U}), 2\left\|\left(T-Z_{0}\right) P\right\|=\left\|\mathscr{D}_{T P} \mid \mathfrak{U} P\right\|$, for every central projection $P$ of $\mathscr{U}$.

Now we prove uniqueness of $Z_{0}$. If $\mathscr{U}_{1}$ is the type I algebra containing $\mathscr{U}$ as

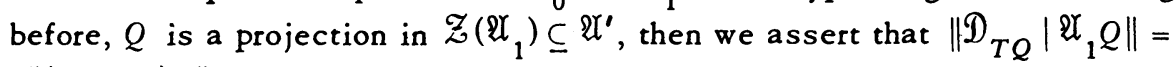
$2\left\|\left(T-Z_{0}\right) Q\right\|$.

Let $P$ be the central support of $Q$ in $\mathcal{Z}(\mathscr{U})$. Then for every $A$ in $\mathscr{U},\|A P\|=$ $\|A Q\|[2]$.

Now,

$$
\left\|\mathscr{D}_{T P}\left|\mathfrak{Q} P\|=2\|\left(T-Z_{0}\right) P\|=2\|\left(T-Z_{0}\right) Q\|\geq\| \mathscr{I}_{T Q}\right| \mathscr{U}_{1} Q\right\| .
$$

On the other hand, if $X$ is in $\mathfrak{A}$,

$$
\begin{aligned}
\left\|\mathscr{D}_{T}(X) P\right\| & =\left\|\mathscr{D}_{T P}(X P)\right\|=\left\|\mathcal{D}_{T}(X) Q\right\|=\left\|\mathscr{D}_{T Q}(X Q)\right\| \\
& \leq\left\|\mathscr{D}_{T Q}\left|\mathscr{Q}_{1} Q\|\| X Q\|=\| \mathscr{D}_{T Q}\right| \mathscr{Q}_{1} Q\right\|\|X P\| .
\end{aligned}
$$

Now Corollary 1 proves the uniqueness of $Z_{0}$.

\section{BIBLIOGRAPHY}

1. J. Diximier, Les algèbres d'opérateurs dans l'espace hilbertien (Algèbres de von Neumann), Cahiers Scientifiques, fasc. 25, Gauthier-Villars, Paris, 1957. MR $20 \sharp 1234$.

2. R. V. Kadison, Unitary invariants for representations of operator algebras, Ann. of Math. (2) 66 (1957), 304-379. MR 19, 665.

3. - Derivations of operator algebras, Ann. of Math. (2) 83 (1966), 280-293. MR $33 \# 1747$.

4. R. V. Kadison, E. C. Lance and J. R. Ringrose, Derivations and automorphisms of operator algebras. II, J. Functional Analysis 1 (1967), 204-221. MR 35 \#5954.

5. R. V. Kadison and J. R. Ringrose, Derivations and automorphisms of operator algebras, Comm. Math. Phys. 4 (1967), 32-63. MR 34 \#6552.

6. M. A. Naimark and S. V. Fomin, Continuous direct sums of Hilbert spaces and some of their applications, Uspehi Mat. Nauk 10 (1955), no. 2 (64), 111-143; English transl., Amer. Math. Soc. Transl. (2) 5 (1957), 35-65. MR 17, 65; MR 18, 748.

7. S. Sakai, Derivations of $\mathbb{W}^{*}$-algebras, Ann. of Math. (2) 83 (1966), 273-279. MR $33 \# 1748$.

8. J. G. Stampfli, The norm of a derivation, Pacific J. Math. 33 (1970), 737-747. MR $42 \# 861$.

TATA INSTITUTE OF FUNDAMENTAL RESEARCH, BOMBAY S, INDIA

Current address: Department of Mathematics, University of Toronto, Toronto, Ontario, Canada 